\title{
The fate of trace organic contaminants during anaerobic digestion of primary sludge: a pilot scale study
}

Revised manuscript submitted to Bioresource Technology

February 2018

Hop V. Phan ${ }^{\mathrm{a}}$, Richard Wickham ${ }^{\mathrm{a}}$, Sihuang Xie ${ }^{\mathrm{a}}$, James A. McDonald ${ }^{\mathrm{b}}$, Stuart J. Khan ${ }^{\mathrm{b}}$, Hao H. Ngo ${ }^{\mathrm{c}}$, Wenshan $\mathrm{Guo}^{\mathrm{c}}$, and Long D. Nghiem ${ }^{\mathrm{c} *}$

${ }^{\text {a }}$ Strategic Water Infrastructure Laboratory, School of Civil, Mining and Environmental Engineering, University of Wollongong, NSW 2522, Australia.

${ }^{\mathrm{b}}$ School of Civil and Environmental Engineering, University of New South Wales, NSW 2052, Australia.

${ }^{c}$ Centre for Technology in Water and Wastewater, School of Civil and Environmental Engineering, University of Technology Sydney, Sydney, NSW 2007, Australia

${ }^{*}$ Corresponding author

Email: duclong.nghiem@uts.edu.au; Ph: +61 (2) 95142625 


\begin{abstract}
A pilot-scale study was conducted to investigate the fate of trace organic contaminants (TrOCs) during anaerobic digestion of primary sludge. Of the 44 TrOCs monitored, 24 were detected in all primary sludge samples. Phase distribution of TrOCs was correlated well with their hydrophobicity ( $>67 \%$ mass in the solid phase when $\operatorname{LogD}>1.5)$. The pilot-scale anaerobic digester achieved a steady performance with a specific methane yield of $0.39-0.92 \mathrm{~L} / \mathrm{gVS}_{\text {removed }}$ and methane composition of $63-65 \%$ despite considerable variation in the primary sludge. The fate of TrOCs in the aqueous and solid phases was governed by their physicochemical properties. Biotransformation was significant $(>83 \%$ ) for five TrOCs with $\log \mathrm{D}<1.5$ and electron donating functional groups in molecular structure. The remaining TrOCs with $\log \mathrm{D}<1.5$ were persistent and thus accumulated in the aqueous phase. Most TrOCs with $\log \mathrm{D}>1.5$ were poorly removed under anaerobic conditions. Sorption onto the solid phase appears to impede the biodegradation of these TrOCs.
\end{abstract}

Keywords: Anaerobic digestion; Pilot-scale; Trace organic contaminants (TrOCs); Primary sludge; Hydrophobicity.

\title{
1. Introduction
}

Anaerobic digestion is the process where microorganisms break down organic materials such as sewage sludge in the absence of oxygen into biogas and digestate. It is a pragmatic platform to facilitate the recovery of both bioenergy and phosphorus from sewage sludge (Christodoulou \& Stamatelatou, 2016; Nghiem et al., 2017; Tuyet et al., 2016). In addition to the economic benefits, environmental and human health protection (i.e. safe management of the solid residuals 
from anaerobic digestion) is equally important. In this context, the ubiquitous occurrence of trace organic contaminants (TrOCs) in municipal wastewater and sewage sludge has emerged as a considerable risk factor associated with biosolids reuse for agricultural production (Luo et al., 2014).

Some TrOCs have been detected in biosolids with concentrations ranging from $\mu \mathrm{g}$ to $\mathrm{mg}$ per $\mathrm{kg}$ of dry sludge (Stasinakis, 2012; Verlicchi \& Zambello, 2015). These include household chemicals and personal care products (e.g. triclosan and triclocarban) and pharmaceuticals (e.g. carbamazepine and diclofenac). These TrOCs are constantly released into sewers, variably persistent to wastewater treatment and some have high affinity to sludge. The occurrence of these TrOCs in biosolids has also manifested in their presence in the biosolids-amended soil (Verlicchi \& Zambello, 2015) and they can bioaccumulate in agricultural crops and may trigger genotoxicity effect (Gonzalez-Gil et al., 2016; Wu et al., 2010).

The removal of TrOCs by aerobic activated sludge treatment has been extensively investigated in recent years (Boonyaroj et al., 2012; Luo et al., 2014; Navaratna et al., 2016). Particularly, more studies have focused on the application of aerobic membrane bioreactor as barrier for TrOC elimination in sewage treatment system (Luo et al., 2017; Phan et al., 2014). Aerobic conditions are generally more effective for TrOC removal. Nevertheless, several compounds, such as sulfur/nitrogen-bearing TrOCs and halogenated aromatic TrOCs have been shown to be more effectively removed under anaerobic condition (Wijekoon et al., 2015). It has been speculated that the sorption of TrOCs into the solid phase can hinder their degradation during anaerobic treatment (Ghattas et al., 2017). An improved understanding of the fate of TrOCs during anaerobic digestion is important to limit the release of TrOCs from wastewater treatment into the 
environment. Indeed, little is known about the removal of TrOCs by anaerobic digestion, particularly under a realistic operational condition.

Studies to date on the removal of TrOCs by anaerobic digestion are mostly in laboratory scale. Although consistent observations have been reported for readily biodegradable compounds (e.g. paracetamol, caffeine, naproxen), significant variation and/or contradictory results have also been reported for persistent TrOCs (e.g. diclofenac, carbamazepine, triclosan, bisphenol A) (Carballa et al., 2007; Malmborg \& Magnér, 2015; Yang et al., 2016; Yang et al., 2017). Most hydrophobic and persistent TrOCs were not biodegraded and remained in the solid residuals (Carballa et al., 2007; Yang et al., 2016). The hydrophobicity of TrOCs may relate to their persistence during anaerobic digestion (Malmborg \& Magnér, 2015; Yang et al., 2017). In addition to hydrophobicity, there can be other sorption mechanisms (such as electrostatic interaction) for TrOCs to partition into the solid phase (Stevens-Garmon et al., 2011).

The role of the intrinsic properties of TrOCs on their biodegradation has been discussed in the literature (Ghattas et al., 2017; Wijekoon et al., 2013; Yang et al., 2017), however, it is still difficult to predict anaerobic biotransformation of TrOCs. This difficulty can be attributed to the low concentration and the structural complexity of TrOCs, as well as the complexity of environmental conditions (e.g. matrix, biodegradable dissolved organic compounds, and $\mathrm{pH}$ ). Moreover, the microorganisms and their enzymatic profiles under specific anaerobic conditions largely depend on the availability of electron acceptors and their respective energy yield.

In most laboratory-scale experiments, TrOCs are spiked into the feed to obtain a sufficiently high concentration under a well-regulated condition. Thus, it is not certain if the observed mechanisms of TrOC removal in the laboratory scale can be applied to a realistic situation where TrOCs occur at a lower concentration and with considerable temporal variations in both 
operating conditions and TrOC concentration in the feed. Indeed, there have been some striking differences in the observed biodegradability between lab- and full-scale studies, particularly for persistent TrOCs (e.g. carbamazepine, diclofenac, and ibuprofen) (Bu et al., 2016). Higher removal of TrOCs has been reported for lab-scale anaerobic digestion (Semblante et al., 2015). To date, very few surveys on TrOC removal have been reported from full-scale anaerobic digestion facilities (Narumiya et al., 2013; Samaras et al., 2013). Due to variations in operating conditions and geographical locations, findings from full-scale surveys are very useful and practical but are limited in generalizability. In this context, a pilot-scale study can facilitate the extrapolation of the current knowledge from the lab to the field by representing a realistic condition and at the same time allowing for a systematic and accurate assessment of the fate of TrOCs during anaerobic digestion.

To date there has not yet been any pilot-scale study to investigate the fate TrOCs during anaerobic digestion. Thus, this study aims to investigate the TrOC behavior during anaerobic digestion using a state-of-the-art pilot-scale system. The occurrence and distribution between the aqueous and solid phases of the 44 TrOCs were systematically examined.

\section{Materials and Methods}

\subsection{Pilot-scale anaerobic digester operation}

A pilot-scale anaerobic digestion system was constructed and installed at a wastewater treatment plant in Australia (Supplementary data). The system consisted of a 1,000 L cylindrical stainless steel reactor and a $100 \mathrm{~L}$ feed tank. The external surface of the reactor was wrapped with a water jacket to maintain a constant mesophilic condition of $35 \pm 1{ }^{\circ} \mathrm{C}$. The reactor was fully enclosed with insulating material to minimize heat loss. A recirculation pump ( $25 \mathrm{~L} / \mathrm{min})$ was operated continuously to mix the reactor. The anaerobic digester was equipped with a supervisory control 
and data acquisition (SCADA) system, and could be remotely controlled. The pilot-scale anaerobic digester was inoculated with anaerobically digested sludge $(600 \mathrm{~L})$ from a full-scale digester at a wastewater treatment plant. The system was operated at hydraulic retention time (HRT) of 20 days. Digestate discharge and feeding occurred automatically four times each day. Each discharge/feeding cycle was approximately 10 minutes, 7.5 L of digestate was withdrawn from the pilot plant and 7.5 L of primary sludge was then introduced into the digester.

\subsection{Digester performance monitoring}

Biogas production and temperature were continuously recorded via the SCADA system. Biogas composition was analyzed using a portable gas analyzer (GA5000 gas analyzer, Geotechnical Instruments Ltd, UK). $\mathrm{pH}$ measurement was conducted using a portable $\mathrm{pH}$ meter (Thermo Scientific, Australia). Total solid (TS), volatile solid (VS), alkalinity and total organic acid (TOA) were measured in accordance with standard methods. Soluble chemical oxygen demand (sCOD) and total chemical oxygen demand (tCOD) were quantified following the US-EPA Standard Method 8000 using high range COD vials (HACH, USA). The sludge samples were centrifuged (3,200 g for 10 min using Allegra X-12R centrifuge, Beckman Coulter, Australia) and then filtered $(1 \mu \mathrm{m}$ filter paper, Filtech, Australia) to obtain the supernatant for sCOD measurement.

\subsection{Trace organic contaminant analysis}

Weekly samples of primary sludge and digestate were collected for TrOC analysis. Each sample was centrifuged at 3,200 g for 10 minutes (Allegra X-12R centrifuge, Beckman Coulter, Australia). The supernatant and pellet were collected for TrOC quantification in the aqueous and solid phases, respectively. The supernatant $(50 \mathrm{~mL})$ was further filtered through $0.7 \mu \mathrm{m}$ filter paper (Filtech, Australia) and then diluted with Milli-Q water into $500 \mathrm{~mL}$. The pellet was 
freeze-dried for $24 \mathrm{~h}$ using an Alpha 1-2 LD plus Freeze Dryer (Christ GmbH, Germany). The dried sludge $(0.5 \mathrm{~g})$ was ground into the powder and subjected to successively ultrasonic solvent extraction with $5 \mathrm{~mL}$ methanol and $5 \mathrm{~mL}$ dichloromethane - methanol $(1: 1 \mathrm{v} / \mathrm{v})$. The solvent was evaporated under nitrogen gas and the extract was reconstituted into $500 \mathrm{~mL}$ with Milli-Q water. The detail of solvent extraction method can be found in Wijekoon et al. (2013). Both aqueous and solid samples were analyzed using the analytical method previously described in Phan et al. (2015).

A total of 44 TrOCs frequently reported in municipal sewage were monitored in this study (Supplementary data). These compounds were chosen based on their environmentally relevant concentrations and represent a diverse range of physical and chemical properties. A surrogate standard $(50 \mu \mathrm{L})$ containing 44 isotopically labeled compounds (corresponding to the 44 selected TrOCs in this study) was introduced into all samples for method recovery and quantification. The samples were then loaded onto the solid phase extraction (SPE) cartridges (6cc/500 HLB cartridges, Waters Australia) successively conditioned with $5 \mathrm{~mL}$ methyl tert-butyl ether, $5 \mathrm{~mL}$ methanol, and 2 x $5 \mathrm{~mL}$ Mili-Q water. The analytes were eluted ethanol $(1 \mathrm{~mL})$. The analysis was performed using a gas chromatography tandem mass spectrometry (GC-MS/MS).

It is noted that, in this study, TrOC removal was measured as the disappearance of the parent compounds, which does not necessary indicate their complete mineralization. Degradation byproducts may be formed during anaerobic digestion. Nevertheless, they are not expected to be toxic. Since biomass from the anaerobic digester is very complex, a detailed characterization of these potential degradation by-products is beyond the scope of our study. 


\subsection{TrOC mass balance}

The mass balance of each TrOC in primary sludge and digestate was calculated as described in Yang et al. (2017):

$C_{\text {in }}=X_{\text {in }} \times T S_{\text {in }}+S_{\text {in }}$

Where $\mathrm{C}_{\mathrm{in}}$ is the total inlet concentration $(\mathrm{ng} / \mathrm{L}), \mathrm{X}_{\mathrm{in}}$ is TrOC concentration in the solid phase of primary sludge (ng/g dry sludge), $\mathrm{TS}_{\mathrm{in}}$ is the total solid concentration of primary sludge $(\mathrm{g} / \mathrm{L})$, and $\mathrm{S}_{\mathrm{in}}$ is the TrOC concentration in the aqueous phase of primary sludge (ng/L).

$C_{\text {out }}=X_{\text {out }} \times T S_{\text {out }}+S_{\text {out }}$

Where $\mathrm{C}_{\text {out }}$ is the total outlet concentration $(\mathrm{ng} / \mathrm{L}), \mathrm{X}_{\text {out }}$ is the TrOC concentration in the solid phase of digestate (ng/g dry sludge), $\mathrm{TS}_{\text {out }}$ is the total solid concentration of wasted digestate $(\mathrm{g} / \mathrm{L})$ and $\mathrm{S}_{\text {out }}$ is the TrOC concentration in the aqueous phase of digestate $(\mathrm{ng} / \mathrm{L})$.

Hence, the mass balance of TrOCs during anaerobic digestion can be presented as:

$C_{\text {in }}=C_{\text {out }}+C_{\text {bio }}$

Where $\mathrm{C}_{\mathrm{bio}}$ is the biotransformated portion of TrOC.

\section{Results and discussion}

\subsection{Occurrence of TrOCs in primary sludge}

The distributions of TrOCs between the solid and aqueous phases in primary sludge were examined by normalizing the concentration to absolute mass per liter of sludge. Of the $44 \mathrm{TrOCs}$ monitored in this study, 24 compounds were detected in all primary sludge samples in both the solid and aqueous phases. A considerable temporal variation in TrOC concentration was 
observed in primary sludge collected weekly from a full-scale wastewater treatment plant (Fig.

$1)$.
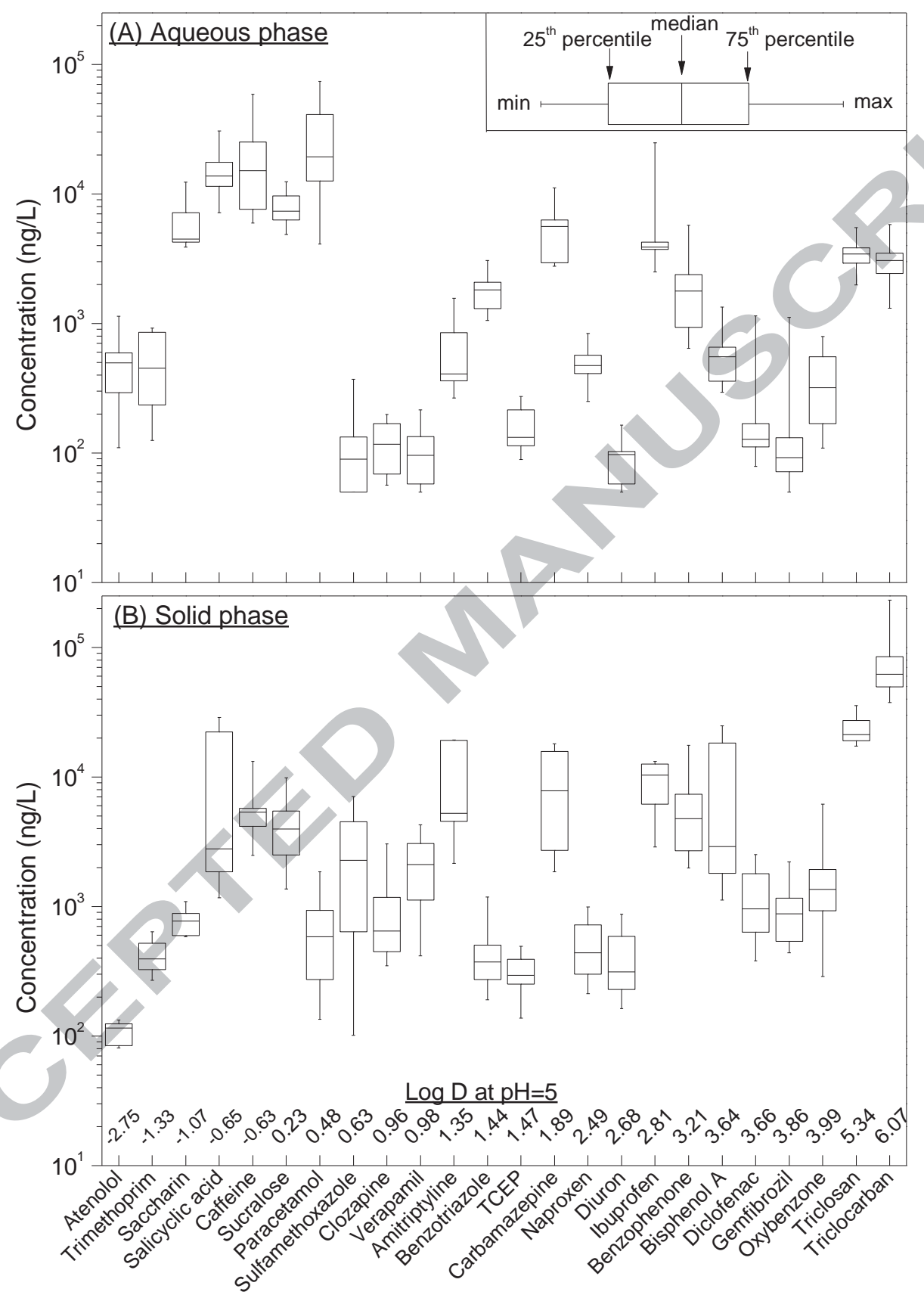

Figure 1: TrOC concentration in primary sludge in the (A) aqueous and (B) solid phase.

Duplicate samples were collected weekly for seven weeks. Concentrations in the solid phase (ng/g) were converted to $\mathrm{ng} / \mathrm{L}$ by multiplying with corresponding total solids $(\mathrm{g} / \mathrm{L})$. TrOCs are 
listed in the order of $\log \mathrm{D}($ at $\mathrm{pH}=5)$. Values of $\log \mathrm{D}$ at $\mathrm{pH}=5$ were obtained from the SciFinder Scholar (ACS) database.

Seven TrOCs (triclocarban, paracetamol, triclosan, caffeine, salicyclic acid, ibuprofen and carbamazepine) exhibited a high concentration in primary sludge $(14-90 \mu \mathrm{g} / \mathrm{L})$. With the exception of salicyclic acid, the occurrence of these TrOCs in primary sludge (Narumiya et al., 2013; Yang et al., 2017) and biosolids (Clarke \& Smith, 2011) have also been reported in the literature. In comparison with raw wastewater (Phan et al., 2015), the primary sludge in this study showed a higher concentration of highly hydrophobic compounds (e.g. triclosan and triclocarban) and a lower concentration of hydrophilic compounds (e.g. caffeine and paracetamol). This is consistent with phase distribution of TrOCs and a high solid content of the primary sludge as discussed below.

The occurrence of triclocarban and triclosan in primary sludge can be attributed to their common application as antimicrobial agents in personal care products (Kookana et al., 2011). In particular, the high concentration of paracetamol in primary sludge is associated with its widespread consumption in Australia. Similarly, the ubiquity of caffeine can be expected as it is commonly found in heavily consumed products such as coffee and tea (Phan et al., 2015). Ibuprofen and carbamazepine are common anti-inflammatory and antiepileptic drugs, respectively, which can be excreted via human urine and then released into sewage system. Salicyclic acid is a phenolic compound, an active form of the most popular pain killer acetylsalicylic acid. It is also a metabolite of keratolytic, dermatic, and food preservative (Ternes, 1998).

Phase distribution of TrOCs was well correlated with hydrophobicity (Fig. 2). TrOCs with $\log D$ from 1.5 (TCEP) were mostly distributed in the solid phase ( $>67 \%$ of total content). Taking into 
account the high TS of primary sludge $(20 \pm 7 \mathrm{~g} / \mathrm{L}, \mathrm{n}=6)$, TrOC content in the primary sludge feed was mostly in the solid phase. Of all 24 TrOCs ubiquitously detected in primary sludge, the mass in the solid phase accounted for more than 50\% total mass for 16 compounds, $20-45 \%$ for 5 compounds and less than $20 \%$ for only 3 compounds. The most prevalent TrOCs in solid phase were triclocarban $(87 \pm 65 \mu \mathrm{g} / \mathrm{L}, \mathrm{n}=7)$ and triclosan $(24 \pm 6 \mu \mathrm{g} / \mathrm{L}, \mathrm{n}=7)$, with solid phase contribution of $97 \%$ and $88 \%$, respectively. These are also the two most hydrophobic compounds investigated in this study. In contrast, two hydrophilic compounds with the highest occurrence in the aqueous phase are paracetamol $(29 \pm 23 \mu \mathrm{g} / \mathrm{L}, \mathrm{n}=7)$ and caffeine $(21 \pm 17 \mu \mathrm{g} / \mathrm{L}, \mathrm{n}=7)$ with solid phase contribution of 2 and $22 \%$, respectively. It is noteworthy that primary sludge $\mathrm{pH}$ was mildly acidic ( $\mathrm{pH}=4.9 \pm 0.7, \mathrm{n}=6)$. The $\log D$ of some compounds changed with increasing $\mathrm{pH}$ during anaerobic digestion ( $\mathrm{pH}$ of $7.0 \pm 0.2, \mathrm{n}=6$ ), due to changes in fractions of neutral and charged species. The change in hydrophobicity led to the different partitioning of TrOCs between solid and aqueous phases as discussed in Section 3.3. 


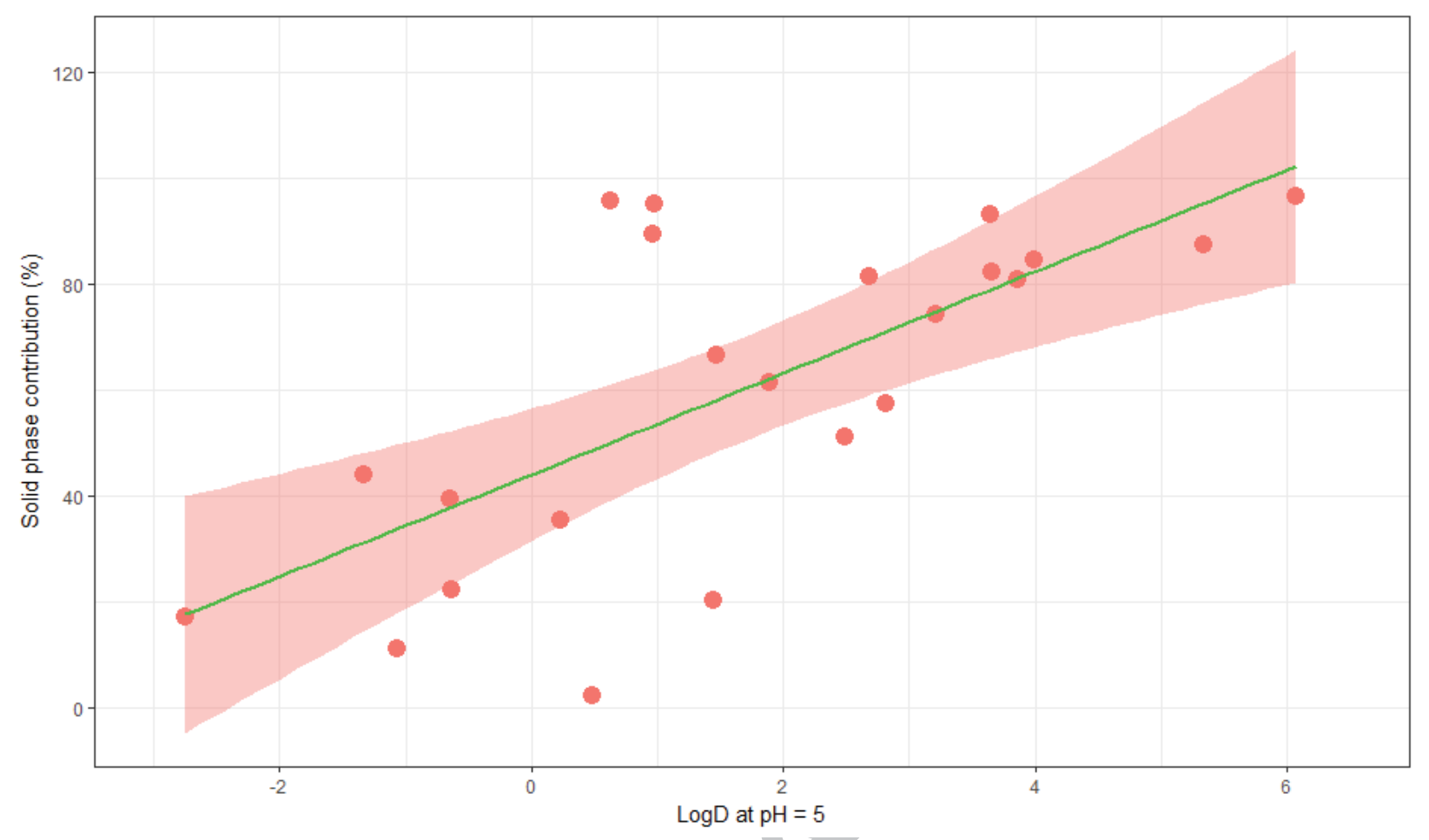

Figure 2: The correlation between $\operatorname{LogD}(\mathrm{pH}=5)$ and the solid phase contribution to the TrOC content in primary sludge. Pearson correlation $=0.70$ and $p$ value $<0.0005$. The shaded area represents the $95 \%$ confident interval.

\subsection{Anaerobic digester performance}

The pilot-scale anaerobic digestion plant was acclimatized for 60 days (approximately 3 times the HRT value) before performance evaluation for over 7 weeks. The anaerobic digester was stable and achieved a methane yield of $0.58 \pm 0.24 \mathrm{~L} \mathrm{CH}_{4} / \mathrm{g} \mathrm{VS}_{\text {removed }}(\mathrm{n}=6)$ over the entire experimental period. Methane yield is a function of methanogenic activity; hence, it is expected to be constant. However, due to significant variation of organic content of the feed primary sludge and the fact that methane yield is calculated based on VS, notable variation in methane yield could be observed over the experimental period. Thus, the observed variation in methane yield can be explained by the fluctuation of organic content in primary sludge, such as tCOD and TS (Fig. 3A \& B). 

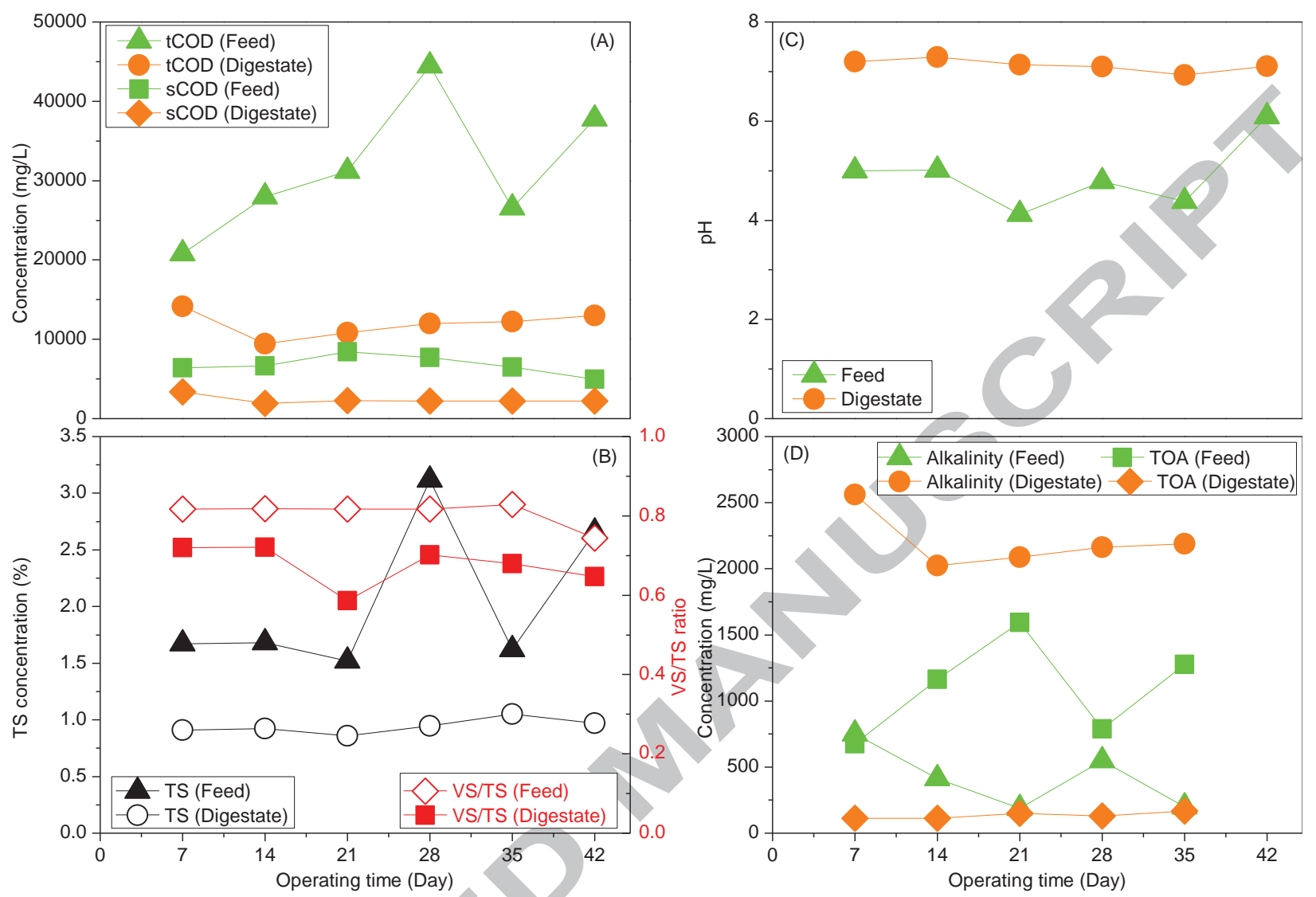

Figure 3: Performance and operational parameters of pilot-scale anaerobic digester in terms of (A) COD concentration in the feed and digestate, (B) TS concentration and VS/TS ratio in the feed and digestate, (C) $\mathrm{pH}$ in the feed and digestate, and (D) alkalinity and TOA in the feed and digestate. Feed was primary sludge collected from a full-scale sewage treatment plant.

During the entire experimental period, the biogas composition was constant with the methane content of $63-65 \%$ and carbon dioxide content of $37-35 \%$. The digestate properties were stable indicating a steady state of anaerobic digestion operation despite notable variation in the feed primary sludge. No evidence of volatile fatty acid or ammonia accumulation in the digester could be observed as demonstrated by a stable and near neutral $\mathrm{pH}(7.1 \pm 0.1)$, high alkalinity content $\left(2205 \pm 210 \mathrm{mg} \mathrm{CaCO}_{3} / \mathrm{L}\right)$, as well as low TOA content $(135 \pm 24 \mathrm{mg} / \mathrm{L})$ in the digestate 
(Fig. $3 C \& D$ ). The biomass of the digestate had a relatively constant VS/TS ratio of $0.7 \pm 0.1$. In comparison to the primary sludge TS $(20 \pm 7 \mathrm{~g} / \mathrm{L})$, solid reduction of $50 \pm 13 \%$ was observed due to anaerobic digestion (Fig. 3B). In addition, the removal of soluble COD was $64 \pm 10 \%$. Overall, results show that the pilot-scale anaerobic digestion plant had a good and steady performance throughout the experimental period.

\subsection{TrOC removal from aqueous and solid phases}

Overall, the TrOC concentration of digestate was stable in both aqueous and solid phases regardless of their concentration variation in the feed (Fig. 4). In contrast, the anaerobic digester showed significant variation in the behavior of different TrOCs for both phases, indicating the governing role of intrinsic TrOC properties. From phase distribution of TrOCs discussed in Section 3.1, $\log D(\mathrm{pH}=7)=1.5($ TCEP $)$ was arbitrarily selected for distinguishing between hydrophilic $(\log \mathrm{D}<1.5)$ and hydrophobic $(\log \mathrm{D}>1.5)$ compounds. 


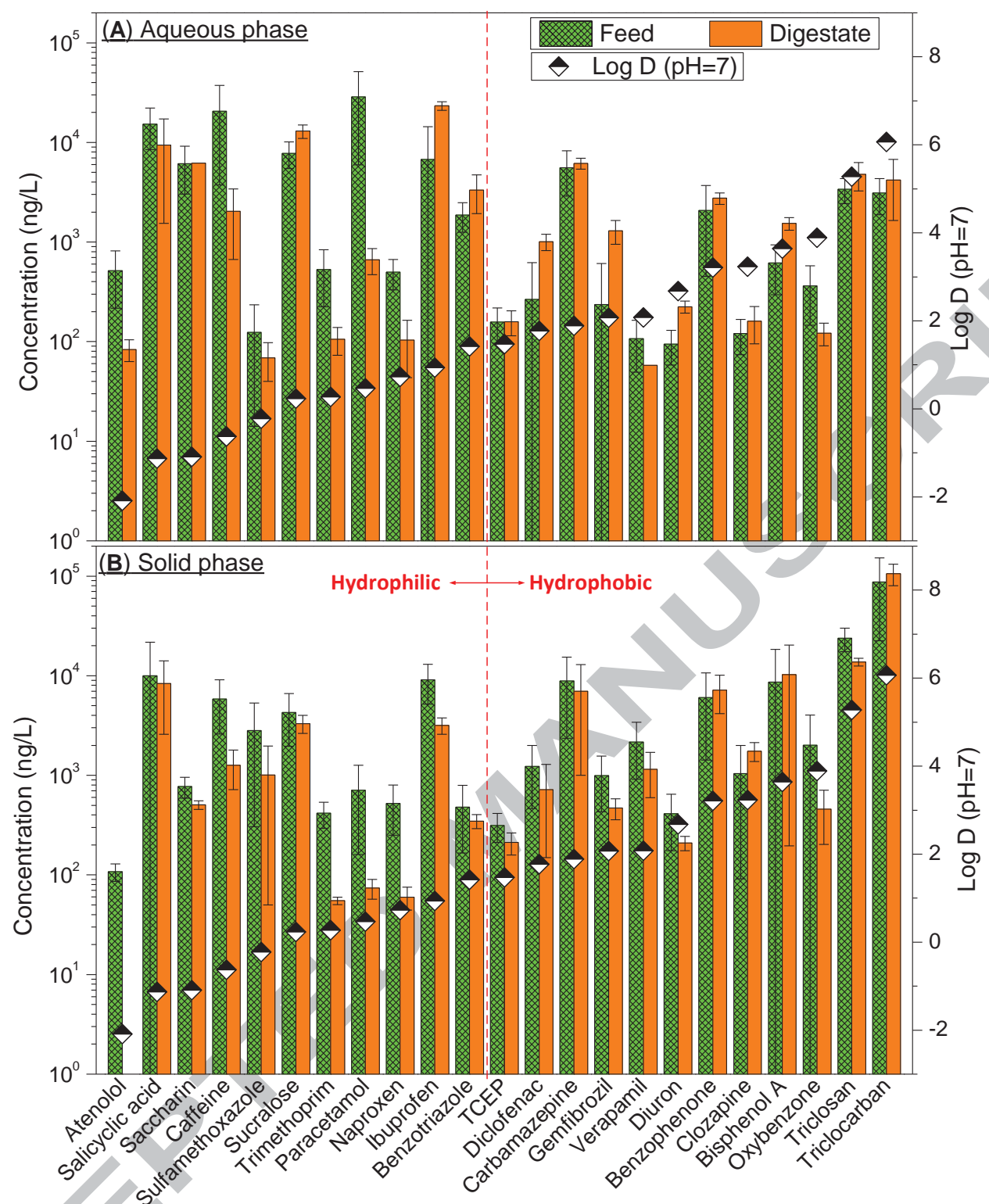

Figure 4: TrOC concentrations in the (A) aqueous and (B) solid phase in the feed (primary sludge) and digestate (anaerobic digester). Error bar represents the standard deviation of weekly duplicate samples collected during 7 weeks. Concentrations in solid phases (ng/g dry sludge) were converted to $\mathrm{ng} / \mathrm{L}$ by multiplying with corresponding total solid values $(\mathrm{g} / \mathrm{L})$. Values of $\operatorname{LogD}$ at $\mathrm{pH}=7$ were obtained from the SciFinder Scholar (ACS) database.

\subsubsection{Hydrophilic compounds}


The removals of the 11 detectable hydrophilic compounds can be divided into three groups. The first group includes five compounds namely atenolol, caffeine, trimethoprim, paracetamol, and naproxen, which were well removed (> 83\%) from both the aqueous and solid phases (Fig. 4). Similar behavior during the anaerobic digestion treatment has been reported for these TrOCs with the exception of atenolol (Narumiya et al., 2013; Yang et al., 2017). The removal of atenolol has only been reported in a lab-scale anaerobic digester at a much lower efficiency (69\%). High removal by anaerobic membrane bioreactor (MBR) for these compounds has also been reported by Wijekoon et al., (2015). These TrOCs have only electron donating functional groups (EDGs) (e.g. $-\mathrm{NH}_{2},-\mathrm{OH},-\mathrm{CH}_{3},-\mathrm{OCH}_{3}$ ) in their molecular structure (Supplementary data) that render them more susceptible to biodegradation. It is noted that biodegradation pathways under anaerobic conditions are diverse and depend on the availability of different types of electron acceptors, both inorganic (e.g. $\mathrm{NO}_{3}, \mathrm{SO}_{4}{ }^{2-}, \mathrm{Fe}^{3+}$, and $\mathrm{Mn}^{4+}$ ) and organic (e.g. dimethyl sulfoxide, dimethyl sulfide). For example, trimethoprim consists of two aromatic rings, a double aminated pyrimidine ring and a phenyl-trimethyl ether. The anaerobic biodegradation pathways of trimethoprim can be activated through phenyl-methyl ether cleavage or via a substitution of pyrimidine ring, depending on the specific conditions (Ghattas et al., 2017).

Two hydrophilic compounds (salicyclic acid and sulfamethoxazole) were moderately removed from both the aqueous and solid phases. The removals of sulfamethoxazole from the aqueous and solid phases were 45 and 64\%, respectively. Anaerobic biodegradation of sulfamethoxazole has been observed under different reducing conditions (e.g. iron, sulfate and methanogenic) (Ghattas et al., 2017; Jia et al., 2017). The anaerobic biodegradation of sulfamethoxazole could be attributed to the presence of only EDGs $\left(-\mathrm{NH}_{2},-\mathrm{CH}_{3}\right)$ in their molecular structure (Supplementary data). While the proposed pathway of sulfamethoxazole biotransformation 
during anaerobic digestion was via the reduction of the electron withdrawing sulfonyl group (Ghattas et al., 2017), the biotransformation by cleavaging the isoxazole ring was observed under iron and sulfate reducing conditions (Jia et al., 2017). Previous studies have reported high removal (> 85\%) of sulfamethoxazole by lab-scale anaerobic MBRs (Monsalvo et al., 2014; Wijekoon et al., 2015) and by anaerobic digesters (Carballa et al., 2007; Narumiya et al., 2013). The moderate removal observed in this study could be attributed to a high solid content in this pilot system. The adsorbed sulfamethoxazole into sludge impeded the biodegradation while the partition into aqueous phase was quickly degraded (Fig. 4). The possible effect of sorption to the solid phase on biodegradation is discussed further in section 3.4.

Salicyclic acid was removed by $39 \%$ and $17 \%$ from the aqueous and solid phase, respectively. Our study appears to be the first to report the removal of salicyclic acid by anaerobic digestion. Salicyclic acid has a strong EDG (-OH) and a moderate electron withdrawing group (EWG) ($\mathrm{COOH}$ ) (Supplementary data). The proposed pathways of salicyclic acid biodegradation under nitrate-reducing conditions were (1) via direct reduction of the aromatic ring, or (2) by reductive ortho-dehydroxylation (Bonting \& Fuchs, 1996). Kesserủ et al. (2005) demonstrated the nitratedependent salicyclic acid degradation under anaerobic condition, in which nitrate reduction provides oxygen for the oxidation of salicylic acid (ring cleavage of catechol).

The remaining hydrophilic TrOCs (saccharin, sucralose, ibuprofen, and benzotriazole) were only removed from the solid phase $(23-65 \%)$. The highest removal from the solid phase was observed for ibuprofen (65\%) (Fig. 4). This behavior of ibuprofen was well explained by its hydrophobicity that significantly decreases according to increasing $\mathrm{pH}$ during anaerobic digestion, from $\log \mathrm{D}=2.8$ at $\mathrm{pH}=5$ in primary sludge to $\log \mathrm{D}=0.9$ at $\mathrm{pH}=7$ in digestate. The substantial accumulation of ibuprofen in aqueous phase indicated its recalcitrance to anaerobic 
degradation. Previous studies have also reported the persistence of ibuprofen under anaerobic conditions (Alvarino et al., 2014; Wijekoon et al., 2015; Yang et al., 2017). Although containing three EDGs in the chemical structure, the branched substitutions on para position of the aromatic ring (Supplementary data) of ibuprofen can possibly interfere with the electron transfer process, and hence, anaerobic degradation (Alvarino et al., 2014).

The anaerobic biodegradation of benzotriazole was possibly initiated via methylation or N-N bond scission at the triazole harboring an EDG (-NH). However, the process was slow and subjected to the available electron acceptors (Liu et al., 2011). Of the two artificial sweeteners, no biodegradation was observed in this study. Sucralose was known for its recalcitrance under both aerobic and anaerobic conditions (Tollefsen et al., 2012). The recalcitrance of sucralose can be explained by a heterocyclic structure carrying three EWGs $(-\mathrm{Cl})$. In term of saccharin, it was well removed during aerobic treatment. While more than $95 \%$ removal of saccharin was observed in the fermentation process, no removal was recorded for digested sewage sludge (Buerge et al., 2011). This variation may be rationalized by the presence of different microbial communities.

\subsubsection{Hydrophobic compounds}

Regarding the twelve hydrophobic TrOCs detected in primary sludge, only oxybenzone and verapamil were biodegradable during anaerobic digestion. They were removed from both the aqueous and solid phases, with average removal efficiencies of 66 and $77 \%$, respectively for oxybenzone, and 46 and 47\%, respectively for verapamil. Oxybenzone removal by anaerobic digestion has not been reported in previous studies. The biodegradable propensity of oxybenzone can be explained by the presence of two EDGs (e.g. $\left.-\mathrm{OH},-\mathrm{OCH}_{3}\right)$ in its molecular structure 
(Supplementary data). During aerobic MBR treatment, more than 90\% removal of oxybenzone has been reported (Wijekoon et al., 2013).

Verapamil has seven methyl groups, which are strong EDGs (Supplementary data), in the molecular structure, thus, it is susceptible to biodegradation. The degradation can be initiated via ether cleavage or N-demethylation. More than $90 \%$ removal by anaerobic MBR was observed for verapamil (Monsalvo et al., 2014). In contrast, no removal from aqueous or solid phase was obtained by a lab-scale anaerobic digester (Yang et al., 2017). A plausible explanation for the variation was the difference of solid content between anaerobic digester and anaerobic MBR. $\operatorname{LogD}$ of verapamil increases from 1.0 at $\mathrm{pH}=5$ to 2.1 at $\mathrm{pH}=7$, resulting in an increase in verapamil sorption into the solid phase. It is possible that sorption into sludge during anaerobic digestion decreased the degradation kinetics of most hydrophobic TrOCs in this study.

Six hydrophobic compounds were only removed from the solid phase at a low efficiency $(22-$ $53 \%$ ). These compounds consist of TCEP, diclofenac, carbamazepine, gemfibrozil, diuron, and triclosan. Amongst these TrOCs, the hydrophobicity of diclofenac and gemfibrozil decreases with increasing $\mathrm{pH}$. The $\log \mathrm{D}$ of diclofenac decreases from 3.7 at $\mathrm{pH}=5$ to 1.8 at $\mathrm{pH}=7$. Similarly, LogD of gemfibrozil reduced from 3.9 to 2.1 with increasing of $\mathrm{pH}$ from 5 to 7 . The decrease of $\operatorname{logD}$ led to the re-partitioning of these two TrOCs from the solid phase into the aqueous phase. Accordingly, the aqueous phase accounted for $59 \%$ and $74 \%$ of the total content in digestate for diclofenac and gemfibrozil, respectively. For moderately hydrophobic compounds (TCEP, carbamazepine, and diuron), similar concentrations were observed between aqueous and solid phase concentrations (Fig. 4).

Although a solid phase removal of $42 \%$ was observed for triclosan, due to a very high hydrophobicity $(\log \mathrm{D}=5.3)$, the solid phase still accounted for more than $74 \%$ of total triclosan 
content in digestate. Previous studies reported a negligible removal of triclosan by anaerobic digester (Narumiya et al., 2013; Yang et al., 2016) while the introduction of sludge shearing in anaerobic digestion with recuperative thickening increase triclosan removal (Yang et al., 2017). Veetil et al. (2012) showed the biotransformation of triclosan into catechol and phenol via a cleavage of the diphenyl bond with subsequent reductive dechlorination under anoxic/anaerobic conditions. Possibly, the presence of phenolic group in ortho position of triclosan chemical structure is relevant for the cleavage of diphenyl ether.

The other hydrophobic TrOCs (benzophenone, clozapine, bisphenol A, and triclocarban) were not removed from either aqueous or solid phases. This result indicated that these TrOCs were persistent under anaerobic conditions. They are all very hydrophobic $(\log \mathrm{D}>3.2$ at $\mathrm{pH}=7)$, and therefore partitioned mainly into sludge (72 - 96\% of the total mass). No transformation of triclocarban under anaerobic condition has been previously observed (Yang et al., 2017). While poor removal of clozapine was reported for lab-scale anaerobic digesters, removal of bisphenol A varied from insignificant to $80 \%$ by different studies (Samaras et al., 2014; Yang et al., 2017). This study appears to be the first to report the removal of benzophenone by anaerobic digestion.

\subsection{Fate of TrOCs during anaerobic digestion}

To further elucidate the fate of TrOCs during anaerobic digestion, mass balances were determined. Their fate from the literature under anaerobic versus aerobic conditions was also compared. The fate of TrOCs during anaerobic digestion was consistent with their behavior in aqueous and solid phase that can be predicted based on their physicochemical properties.

The mass balance revealed that biotransformation was significant for seven compounds, including six hydrophilic TrOCs (atenolol, caffeine, trimethoprim, paracetamol, naproxen and sulfamethoxazole) and one hydrophobic compound (oxybenzone) (Fig. 5). Most studies of 
aerobic treatment demonstrated a good biotransformation of these seven biodegradable TrOCs, except for some variation in the case of trimethoprim and sulfamethoxazole (Luo et al., 2014; Phan et al., 2016; Wijekoon et al., 2013). Compared to aerobic treatment, it is speculated that the negative redox potential can enhance the biodegradation of sulfamethoxazole and trimethoprim (Alvarino et al., 2014; Narumiya et al., 2013; Yang et al., 2017). Xue et al. (2010) reported that the biodegradation kinetic of trimethoprim under anaerobic conditions (particularly nitrate reducing condition) was ten times higher than the one of aerobic conditions. While the presence of pyrimidine can render anaerobic biodegradation of trimethoprim, the sulfonyl group may facilitate the reductive biotransformation of sulfamethoxazole (Alvarino et al., 2014). In addition, Wijekoon et al. (2015) suggested the role of sulfur and nitrogen reducing bacteria on enhancing the removal of these nitrogen/sulfur-bearing compounds under anaerobic conditions.

Five hydrophilic compounds (salicyclic acid, saccharin, sucralose, ibuprofen and benzotriazole) and two hydrophobic compounds (diclofenac and gemfibrozil) were mainly discharged via the aqueous phase during anaerobic digestion (Fig. 5). Notably, ibuprofen and gemfibrozil were well removed under aerobic treatment (Luo et al., 2014; Phan et al., 2016), but highly persistent during anaerobic digestion (Carballa et al., 2007; Malmborg \& Magnér, 2015; Yang et al., 2017). A similar behavior during aerobic and anaerobic biodegradation of these two compounds has been demonstrated in the literature (Conkle et al., 2012; Schmidt et al., 2017). Along with the chemical structure of ibuprofen as mention in Section 3.3.1, the lack of sulfur or nitrogen in both ibuprofen and gemfibrozil molecular structure could be another explanation for their persistence under anaerobic condition (Wijekoon et al., 2015).

Diclofenac was previously reported to be poorly removed under aerobic conditions due to the structure containing a strong EWG (i.e. chloro); its anaerobic degradation varied between 
studies. A survey of a full-scale anaerobic digestion plant showed only $27 \%$ removal of diclofenac (Samaras et al., 2013); whereas, moderate to high removal of diclofenac was obtained in some lab-scale anaerobic digesters (Carballa et al., 2007; Samaras et al., 2014; Yang et al., 2017). The more likely pathway of anaerobic diclofenac biotransformation was reductive dechlorination. The variation in diclofenac biotransformation can be expected since the biological reductive dechlorination was often catalyzed by specialized bacterial species under relatively specific conditions (e.g. electron donor, catalyst or trace element). Other possible pathways of anaerobic diclofenac biotransformation were via a hydroxylation of the benzene ring or a decarboxylation of the phenyl acetate (Ghattas et al., 2017).

The remaining hydrophobic TrOCs were mostly detected in the solid residual. Of these compounds, TCEP, carbamazepine and diuron were well known for their recalcitrance to biodegradation under both aerobic (Luo et al., 2014; Phan et al., 2016) and anaerobic conditions (Alvarino et al., 2014; Malmborg \& Magnér, 2015; Yang et al., 2017). Their recalcitrance can be attributed to the presence of strong EWG (-Cl) in their chemical structure (Tadkaew et al., 2011).

Other hydrophobic TrOCs (verapamil, clozapine, bisphenol A, triclosan and triclocarban) were degraded well under aerobic conditions (Luo et al., 2014; Phan et al., 2016). The high aerobic removal of these TrOCs has been explained by their sorption to the activated sludge that enhanced biological degradation (Tadkaew et al., 2011). The observation under anaerobic condition contradicts that of the aerobic condition and could be explained by differences in biodegradation kinetics, sludge characteristics and microbial population between aerobic and anaerobic processes (Barret et al., 2010; Ghattas et al., 2017; Xue et al., 2010). Sorption onto sludge under anaerobic condition can reduce the bioavailability of TrOCs for microbial degradation. Aemig et al. (2016) observed that the distribution of TrOCs in the sludge particles 
depended not only on the physicochemical properties of TrOCs but also the quantity/quality of the particle organic matter. Particularly, during anaerobic digestion, sorbed TrOCs were relocated toward the less accessible organic fractions, thus, reducing their availability for biodegradation. Yang et al. (2017) demonstrated that sludge shearing application during anaerobic digestion can facilitate the circulation of TrOCs between solid and aqueous phase that increase the biotransformation of some hydrophobic compounds (e.g. TCEP and triclosan).

Future studies are necessary for conclusively validating the effect of sorption on bioavailability of TrOCs during anaerobic digestion by simultaneously considering the sludge characterization, microbial population, biodegradation kinetics of TrOCs and their relationship with anaerobic biological processes (hydrolysis, acidogenesis, acetogenesis and methanogenesis). 


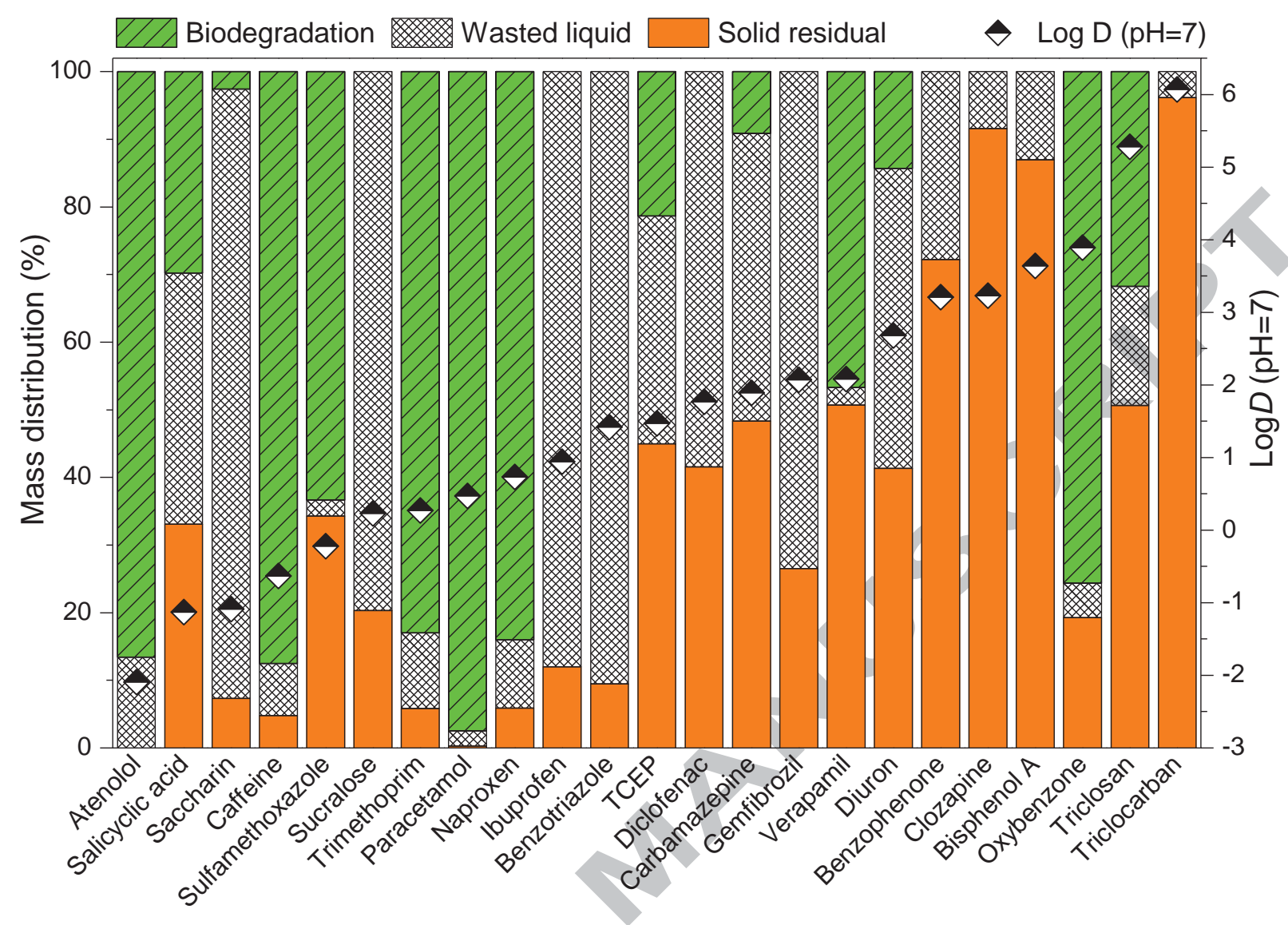

Figure 5: Mass distribution shows the fate of TrOCs during anaerobic digestion at pilot-scale. Values of $\operatorname{LogD}$ at $\mathrm{pH}=7$ were obtained from the SciFinder Scholar (ACS) database. 


\section{Conclusions}

This study profiled 44 TrOCs during stable operation of a pilot-scale anaerobic digester treating primary sludge. In total, 24 compounds were always present in primary sludge; some of them have been reported for the first time. The solid phase accounted for most of the mass of TrOCs in primary sludge. The behavior of TrOCs in the aqueous and solid phases during anaerobic digestion was governed by their physicochemical properties. A high biotransformation rate was observed for hydrophilic TrOCs with electron donating functional groups. Sorption onto sludge under anaerobic conditions is likely to impede the biotransformation of hydrophobic TrOCs.

\section{Supplementary data}

E-supplementary data of this work can be found in online version of the paper.

\section{References}

[1] Aemig, Q., Chéron, C., Delgenès, N., Jimenez, J., Houot, S., Steyer, J.-P., Patureau, D. 2016. Distribution of Polycyclic Aromatic Hydrocarbons (PAHs) in sludge organic matter pools as a driving force of their fate during anaerobic digestion. Waste Manage., 48, 389-396.

[2] Alvarino, T., Suarez, S., Lema, J.M., Omil, F. 2014. Understanding the removal mechanisms of PPCPs and the influence of main technological parameters in anaerobic UASB and aerobic CAS reactors. J. Hazard. Mater., 278, 506-513.

[3] Asimakopoulos, A.G., Ajibola, A., Kannan, K., Thomaidis, N.S. 2013. Occurrence and removal efficiencies of benzotriazoles and benzothiazoles in a wastewater treatment plant in Greece. Sci. Total Environ., 452, 163-171. 
[4] Barret, M., Carrère, H., Latrille, E., Wisniewski, C., Patureau, D. 2010. Micropollutant and Sludge Characterization for Modeling Sorption Equilibria. Environ. Sci. Technol., 44, 11001106.

[5] Bonting, C.F.C., Fuchs, G. 1996. Anaerobic metabolism of 2-hydroxybenzoic acid (salicylic acid) by a denitrifying bacterium. Arch. Microbiol., 165, 402-408.

[6] Boonyaroj, V., Chiemchaisri, C., Chiemchaisri, W., Theepharaksapan, S., Yamamoto, K. 2012. Toxic organic micro-pollutants removal mechanisms in long-term operated membrane bioreactor treating municipal solid waste leachate. Bioresour. Technol., 113, 174-180.

[7] Bu, Q., Shi, X., Yu, G., Huang, J., Wang, B. 2016. Assessing the persistence of pharmaceuticals in the aquatic environment: Challenges and needs. Emerging Contaminants, 2, $145-147$.

[8] Buerge, I.J., Keller, M., Buser, H.-R., Müller, M.D., Poiger, T. 2011. Saccharin and Other Artificial Sweeteners in Soils: Estimated Inputs from Agriculture and Households, Degradation, and Leaching to Groundwater. Environ. Sci. Technol., 45, 615-621.

[9] Carballa, M., Omil, F., Ternes, T., Lema, J.M. 2007. Fate of pharmaceutical and personal care products (PPCPs) during anaerobic digestion of sewage sludge. Water Res., 41, 2139-2150.

[10] Christodoulou, A., Stamatelatou, K. 2016. Overview of legislation on sewage sludge management in developed countries worldwide. Water Science and Technology, 73, 453-462.

[11] Clarke, B.O., Smith, S.R. 2011. Review of 'emerging' organic contaminants in biosolids and assessment of international research priorities for the agricultural use of biosolids. Environ. Int., 37, 226-247. 
[12] Conkle, J.L., Gan, J., Anderson, M.A. 2012. Degradation and sorption of commonly detected PPCPs in wetland sediments under aerobic and anaerobic conditions. J. Soils Sed., 12, 1164-1173.

[13] Ghattas, A.-K., Fischer, F., Wick, A., Ternes, T.A. 2017. Anaerobic biodegradation of (emerging) organic contaminants in the aquatic environment. Water Res., 116, 268-295.

[14] Gonzalez-Gil, L., Papa, M., Feretti, D., Ceretti, E., Mazzoleni, G., Steimberg, N., Pedrazzani, R., Bertanza, G., Lema, J.M., Carballa, M. 2016. Is anaerobic digestion effective for the removal of organic micropollutants and biological activities from sewage sludge? Water Res., 102, 211-220.

[15] Jia, Y., Khanal, S.K., Zhang, H., Chen, G.-H., Lu, H. 2017. Sulfamethoxazole degradation in anaerobic sulfate-reducing bacteria sludge system. Water Res., 119, 12-20.

[16] Kesserü, P., Kiss, I., Bihari, Z., Pál, K., Portörő, P., Polyák, B. 2005. Nitrate-dependent salicylate degradation by Pseudomonas butanovora under anaerobic conditions. Bioresour. Technol., 96, 779-784.

[17] Kookana, R.S., Ying, G.-G., Waller, N.J. 2011. Triclosan: its occurrence, fate and effects in the Australian environment. Water Science and Technology, 63, 598-604.

[18] Liu, Y.-S., Ying, G.-G., Shareef, A., Kookana, R.S. 2011. Biodegradation of three selected benzotriazoles under aerobic and anaerobic conditions. Water Res., 45, 5005-5014.

[19] Luo, W., Phan, H.V., Li, G., Hai, F.I., Price, W.E., Elimelech, M., Nghiem, L.D. 2017. An Osmotic Membrane Bioreactor-Membrane Distillation System for Simultaneous Wastewater Reuse and Seawater Desalination: Performance and Implications. Environ. Sci. Technol., 51, 14311-14320. 
[20] Luo, Y., Guo, W., Ngo, H.H., Nghiem, L.D., Hai, F.I., Zhang, J., Liang, S., Wang, X.C. 2014. A review on the occurrence of micropollutants in the aquatic environment and their fate and removal during wastewater treatment. Sci. Total Environ., 473, 619-641.

[21] Malmborg, J., Magnér, J. 2015. Pharmaceutical residues in sewage sludge: Effect of sanitization and anaerobic digestion. J. Environ. Manage., 153, 1-10.

[22] Monsalvo, V.M., McDonald, J.A., Khan, S.J., Le-Clech, P. 2014. Removal of trace organics by anaerobic membrane bioreactors. Water Res., 49, 103-112.

[23] Narumiya, M., Nakada, N., Yamashita, N., Tanaka, H. 2013. Phase distribution and removal of pharmaceuticals and personal care products during anaerobic sludge digestion. J. Hazard. Mater., 260, 305-312.

[24] Navaratna, D., Shu, L., Jegatheesan, V. 2016. Evaluation of herbicide (persistent pollutant) removal mechanisms through hybrid membrane bioreactors. Bioresour. Technol., 200, 795-803.

[25] Nghiem, L.D., Koch, K., Bolzonella, D., Drewes, J.E. 2017. Full scale co-digestion of wastewater sludge and food waste: Bottlenecks and possibilities. Renewable and Sustainable Energy Reviews, 72, 354-362.

[26] Phan, H.V., Hai, F.I., Kang, J., Dam, H.K., Zhang, R., Price, W.E., Broeckmann, A., Nghiem, L.D. 2014. Simultaneous nitrification/denitrification and trace organic contaminant (TrOC) removal by an anoxic-aerobic membrane bioreactor (MBR). Bioresour. Technol., 165, $96-104$.

[27] Phan, H.V., Hai, F.I., McDonald, J.A., Khan, S.J., Zhang, R., Price, W.E., Broeckmann, A., Nghiem, L.D. 2015. Nutrient and trace organic contaminant removal from wastewater of a resort 
town: Comparison between a pilot and a full scale membrane bioreactor. Int. Biodeterior. Biodegrad., 102, 40-48.

[28] Phan, H.V., McDonald, J.A., Hai, F.I., Price, W.E., Khan, S.J., Fujioka, T., Nghiem, L.D. 2016. Biological performance and trace organic contaminant removal by a side-stream ceramic nanofiltration membrane bioreactor. Int. Biodeterior. Biodegrad., 113, 49-56.

[29] Samaras, V.G., Stasinakis, A.S., Mamais, D., Thomaidis, N.S., Lekkas, T.D. 2013. Fate of selected pharmaceuticals and synthetic endocrine disrupting compounds during wastewater treatment and sludge anaerobic digestion. J. Hazard. Mater., 244, 259-267.

[30] Samaras, V.G., Stasinakis, A.S., Thomaidis, N.S., Mamais, D., Lekkas, T.D. 2014. Fate of selected emerging micropollutants during mesophilic, thermophilic and temperature co-phased anaerobic digestion of sewage sludge. Bioresour. Technol., 162, 365-372.

[31] Schmidt, N., Page, D., Tiehm, A. 2017. Biodegradation of pharmaceuticals and endocrine disruptors with oxygen, nitrate, manganese (IV), iron (III) and sulfate as electron acceptors. Journal of Contaminant Hydrology, 203, 62-69.

[32] Semblante, G.U., Hai, F.I., Huang, X., Ball, A.S., Price, W.E., Nghiem, L.D. 2015. Trace organic contaminants in biosolids: Impact of conventional wastewater and sludge processing technologies and emerging alternatives. J. Hazard. Mater., 300, 1-17.

[33] Stasinakis, A.S. 2012. Review on the fate of emerging contaminants during sludge anaerobic digestion. Bioresour. Technol., 121, 432-440.

[34] Stasinakis, A.S., Thomaidis, N.S., Arvaniti, O.S., Asimakopoulos, A.G., Samaras, V.G., Ajibola, A., Mamais, D., Lekkas, T.D. 2013. Contribution of primary and secondary treatment 
on the removal of benzothiazoles, benzotriazoles, endocrine disruptors, pharmaceuticals and perfluorinated compounds in a sewage treatment plant. Sci. Total Environ., 463, 1067-1075.

[35] Stevens-Garmon, J., Drewes, J.E., Khan, S.J., McDonald, J.A., Dickenson, E.R.V. 2011. Sorption of emerging trace organic compounds onto wastewater sludge solids. Water Res., 45, $3417-3426$.

[36] Subedi, B., Kannan, K. 2014. Fate of Artificial Sweeteners in Wastewater Treatment Plants in New York State, U.S.A. Environ. Sci. Technol., 48, 13668-13674.

[37] Tadkaew, N., Hai, F.I., McDonald, J.A., Khan, S.J., Nghiem, L.D. 2011. Removal of trace organics by MBR treatment: The role of molecular properties. Water Res., 45, 2439-2451.

[38] Ternes, T.A. 1998. Occurrence of drugs in German sewage treatment plants and rivers1Dedicated to Professor Dr. Klaus Haberer on the occasion of his 70th birthday.1. Water Res., 32, 3245-3260.

[39] Tollefsen, K.E., Nizzetto, L., Huggett, D.B. 2012. Presence, fate and effects of the intense sweetener sucralose in the aquatic environment. Sci. Total Environ., 438, 510-516.

[40] Tuyet, N.T., Dan, N.P., Vu, N.C., Trung, N.L.H., Thanh, B.X., De Wever, H., Goemans, M., Diels, L. 2016. Laboratory-scale membrane up-concentration and co-anaerobic digestion for energy recovery from sewage and kitchen waste. Water Science and Technology, 73, 597-606.

[41] Veetil, P., Vijaya Nadaraja, A., Bhasi, A., Khan, S., Bhaskaran, K. 2012. Degradation of Triclosan under Aerobic, Anoxic, and Anaerobic Conditions. Appl. Biochem. Biotechnol., 167, $1603-1612$. 
[42] Verlicchi, P., Zambello, E. 2015. Pharmaceuticals and personal care products in untreated and treated sewage sludge: Occurrence and environmental risk in the case of application on soil — A critical review. Sci. Total Environ., 538, 750-767.

[43] Wijekoon, K.C., Hai, F.I., Kang, J., Price, W.E., Guo, W., Ngo, H.H., Nghiem, L.D. 2013. The fate of pharmaceuticals, steroid hormones, phytoestrogens, UV-filters and pesticides during MBR treatment. Bioresour. Technol., 144, 247-254.

[44] Wijekoon, K.C., McDonald, J.A., Khan, S.J., Hai, F.I., Price, W.E., Nghiem, L.D. 2015. Development of a predictive framework to assess the removal of trace organic chemicals by anaerobic membrane bioreactor. Bioresour. Technol., 189, 391-398.

[45] Wu, C., Spongberg, A.L., Witter, J.D., Fang, M., Czajkowski, K.P. 2010. Uptake of Pharmaceutical and Personal Care Products by Soybean Plants from Soils Applied with Biosolids and Irrigated with Contaminated Water. Environ. Sci. Technol., 44, 6157-6161.

[46] Xue, W., Wu, C., Xiao, K., Huang, X., Zhou, H., Tsuno, H., Tanaka, H. 2010. Elimination and fate of selected micro-organic pollutants in a full-scale anaerobic/anoxic/aerobic process combined with membrane bioreactor for municipal wastewater reclamation. Water Res., 44, 5999-6010.

[47] Yang, S., Hai, F.I., Price, W.E., McDonald, J., Khan, S.J., Nghiem, L.D. 2016. Occurrence of trace organic contaminants in wastewater sludge and their removals by anaerobic digestion. Bioresour. Technol., 210, 153-159.

[48] Yang, S., McDonald, J., Hai, F.I., Price, W.E., Khan, S.J., Nghiem, L.D. 2017. The fate of trace organic contaminants in sewage sludge during recuperative thickening anaerobic digestion. Bioresour. Technol., 240, 197-206. 